\title{
Characterization and performance analysis of generalized BOC modulations for GNSS
}

\author{
Fernando D. Nunes ${ }^{1,2}$ (i) | Fernando M. G. Sousa ${ }^{1,3}$ (i) | José M. N. Leitão ${ }^{1,2}$ (i)
}

${ }^{1}$ Instituto de Telecomunicações, Lisbon, Portugal

${ }^{2}$ Instituto Superior Técnico, Universidade de Lisboa, Lisbon, Portugal

${ }^{3}$ Instituto Superior de Engenharia de Lisboa, Instituto Politécnico de Lisboa, Lisbon, Portugal

\section{Correspondence}

Fernando D. Nunes, Instituto de Telecomunicações, Lisbon, Portugal. Email: nunes@lx.it.pt

\begin{abstract}
Novel modulations, based on the binary chirp waveform, are proposed as generalizations of binary offset carrier (BOC) schemes. They allow enhancement of the performance of Global Navigation Satellite System (GNSS) receivers affected by Gaussian noise, multipath, and interference. Adequate selection of parameters enables efficient use of the channel bandwidth to enlarge the Gabor bandwidth. Schemes characterized by autocorrelations with small secondary peaks are obtainable, permitting minimization of the probability of code acquisition with incorrect code phase and reducing the probability of false code-locks in tracking mode. Increased robustness to multipath and interference also occurs. These advantages make the proposed modulation schemes promising candidates for future navigation signals.
\end{abstract}

\section{1 | INTRODUCTION}

Nowadays, Global Navigation Satellite Systems (GNSS), such as the modernized GPS, Galileo, or BeiDou, use signals based on the binary offset carrier (BOC) modulation to increase the mean squared bandwidth (MSB) without incurring excessive bandwidth expansions. ${ }^{1,2}$ They were designed in such a way that the degree of overlapping of the power spectra with other modulations is minimized in order to decrease the mutual interference. BOC modulations result from multiplying a BPSK-R (Binary Phase Shift Keying Robust) spreading time series with chip duration $T_{c}=T_{c 0} / n$, where $T_{c 0}=10^{-3} / 1023 \mathrm{~s}$, by a square wave subcarrier of frequency $m / T_{c 0} \mathrm{~Hz}$, with $m, n \geq 1$. By varying the phase of the square wave, sine-phased $(\operatorname{BOCs}(m, n))$ or cosine-phased BOC $(\operatorname{BOCc}(m, n))$ modulations are obtained. BOC modulations are characterized by multipeaked autocorrelation functions bounded by a triangular envelope with duration equal to twice the chip interval. For instance, in the case of $\operatorname{BOCs}(q n, n)$ signaling with $n$ and $q$ integers, the number of secondary peaks reaches $2(2 q-1)$. These secondary peaks, provoked by the square wave subcarrier, lead to false code-locks of the delay-locked loop, which are combatted with techniques such as bump-jumping. ${ }^{3,4}$ In addition, the autocorrelation secondary peaks are responsible for the oscillatory nature of multipath error envelopes that occur with large secondary ray delays, thus diminishing the receiver's robustness to multipath. Therefore, modulations with a small number of autocorrelation secondary peaks and secondary peaks with low intensity are highly desirable.

The purpose of this work is to define and analyze new types of modulations that minimize the drawbacks of the BOC schemes. These are obtained by replacing the square wave subcarrier of the BOC modulations with the binary waveform that results from feeding a two-level comparator with a (sinusoidal) chirp signal in the chip interval (binary chirp waveform). In the following text, we call these new modulations generalized binary offset carrier (or GBOC). Previous work by the authors, ${ }^{5}$ has shown that the reduction of amplitude of the autocorrelation secondary peaks is achieved by using a chirp signal with instantaneous frequency changing exponentially with time. The current work builds on the results obtained therein by considering, instead, a chirp signal with linear frequency variation within the chip interval. 


\section{2 | DEFINITION OF THE GBOC SIGNALING}

Consider that, in the chip interval, the square wave of the BOC schemes is replaced by the waveform

$$
y(t)=\operatorname{sign}\{\sin \phi(t)\}, \quad 0 \leq t \leq T_{c},
$$

with the phase given by

$$
\phi(t)=2 p \pi\left[a\left(\frac{t}{T_{c}}\right)+(1-a)\left(\frac{t}{T_{c}}\right)^{2}\right]+\theta, \quad 0 \leq t \leq T_{c},
$$

such that $\phi\left(T_{c}\right)-\phi(0)=2 p \pi, p=1,2, \ldots$ and $0 \leq$ $a \leq 2$. The phase rate $d \phi(t) / d t$ increases in the chip interval for $0 \leq a<1$, decreases for $1<a \leq 2$ and is constant for $a=1$. The case $a=1$ and $\theta=0$ corresponds to the $\operatorname{BOCs}(m, n)$ modulation, where $p=m / n$ is the number of square wave periods in the chip interval of duration $T_{c}=T_{c 0} / n$. Quantities $a=1$ and $\theta=\pi / 2$ generate instead the $\operatorname{BOCc}(m, n)$ modulation. In the following, we define the GBOCs and GBOCc modulations for $0 \leq a \leq 2$, with $\theta$ taking the values 0 and $\pi / 2$, respectively. Figure 1 illustrates a few cases of phase $\phi(t)$ for $\theta=0$ (GBOCs) with $p=5$ (solid curves) and $p=6$ (dashed curves). The curves for GBOCc differ from those in the figure by a vertical shift of $\theta=\pi / 2$.

The GBOCs and GBOCc modulations are completely characterized by the triplet $m, n, a$, and we refer to them as $\operatorname{GBOCs}(m, n, a)$ and $\operatorname{GBOCc}(m, n, a)$. The parameters $m$ and $n$ are related by $m=n p$, with $2 p$ and $2 p+1$ being the number of rectangular pulses in the chip interval for the the GBOCs and GBOCc schemes, respectively. Figure 2 exhibits the waveforms $y(t)$ of GBOCs schemes for $m=2$ and $n=1$ with $a=0$ and $a=0.5$.

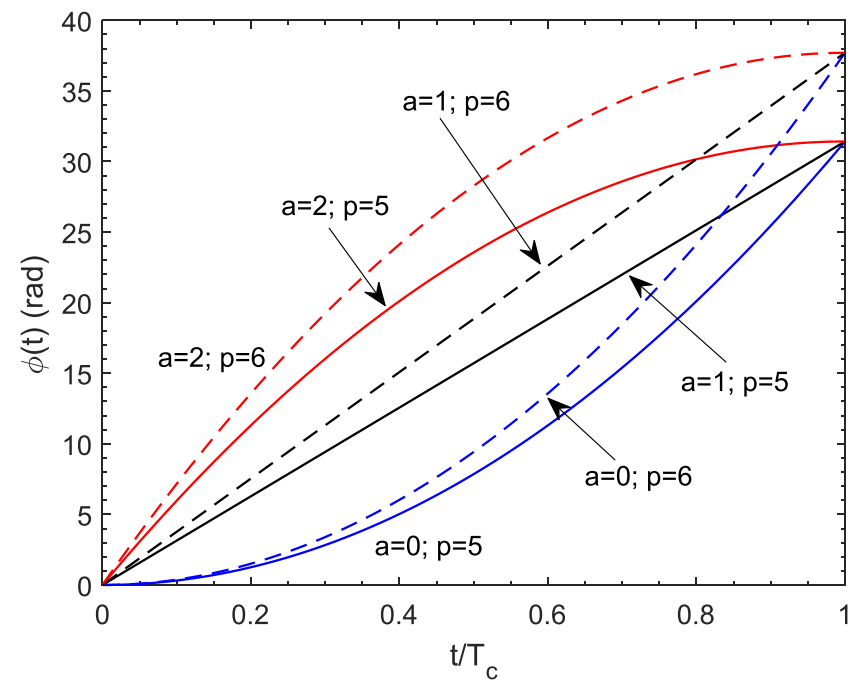

FIGURE 1 Curves of $\phi(t)$ for GBOCs modulation with $p=5$ (solid curves) and $p=6$ (dashed curves) [Color figure can be viewed at wileyonlinelibrary.com and www.ion.org]

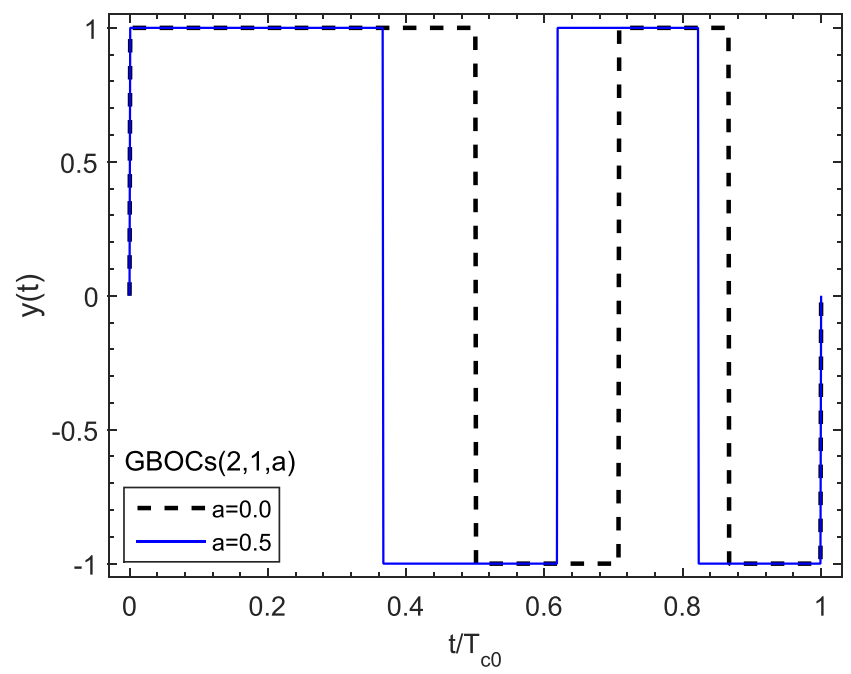

FIGURE 2 Waveforms of $\operatorname{GBOCs}(2,1, a)$ with $a=0$ and $a=0.5$ [Color figure can be viewed at wileyonlinelibrary.com and www.ion.org]

Assume an infinite bandwidth for $y(t)$. For $a \neq 1$, the zero-crossing epochs of $y(t)$ are given by

$$
t_{i}=\frac{T_{c}}{2(1-a)}\left[-a+\sqrt{a^{2}+2(1-a)(i-\theta / \pi) / p}\right],
$$

with $i=0, \ldots, 2 p$, for GBOCs schemes or $i=1, \ldots, 2 p$, for GBOCc schemes. For $a=1$ the zero-crossing epochs are given by $t_{i}=i T_{c} /(2 p)$, with $i=0, \ldots, 2 p$ (BOCs) or $t_{i}=(i-1 / 2) T_{c} /(2 p)$, with $i=1, \ldots, 2 p($ BOCc $)$.

The GBOC signaling waveforms can be expressed as the following sum of rectangular pulses with amplitude levels \pm 1

$$
y(t)=-\sum_{i=1}^{M}(-1)^{i} \Pi\left(\frac{t-\bar{t}_{i}}{T_{i}}\right),
$$

where $\Pi\left(\left(t-\bar{t}_{i}\right) / T_{i}\right)$ denotes a rectangular pulse of height equal to 1 , centered at $t=\bar{t}_{i}$, with duration $T_{i}=t_{i}-t_{i-1}$. In (4), the number of pulses in the chip period is $M=2 p$ for GBOCs schemes, or $M=2 p+1$, for GBOCc schemes, and $\bar{t}_{i}=\left(t_{i-1}+t_{i}\right) / 2, i=1, \ldots, M$, with $t_{0}=0$ and $t_{M}=T_{c}$.

Consider the baseband pulse amplitude modulation signal

$$
s(t)=\sum_{k=0}^{N-1} c_{k} y\left(t-k T_{c}\right),
$$

where $\left\{c_{k}, k=0,1, \ldots, N-1\right\}$, with $c_{k}= \pm 1$, is a binary random sequence of chips, $T_{c}$ is the chip duration, and $y(t)$ is the chip waveform. Let $S(f)$ denote the Fourier transform of $s(t)$. Then

$$
|S(f)|^{2}=|Y(f)|^{2}\left|\sum_{k=0}^{N-1} c_{k} \exp \left(-j 2 \pi f k T_{c}\right)\right|^{2},
$$


where

$$
Y(f)=-\sum_{i=1}^{M}(-1)^{i} T_{i} \operatorname{sinc}\left(f T_{i}\right) \exp \left(-j 2 \pi f \bar{t}_{i}\right)
$$

is the Fourier transform of $y(t)$ and $\operatorname{sinc}(x) \equiv \sin (\pi x) /(\pi x)$. Since the expected value of $|S(f)|^{2}$ is equal to $N|Y(f)|^{2}$, the power spectral density (PSD) of $s(t)$ for random data channels with ideal (infinite period) spreading codes and $N \rightarrow \infty$ is $G_{s}(f)=|Y(f)|^{2} / T_{c}$, according to Betz. ${ }^{6}$ Thus, the PSD of GBOC schemes is given by

$$
\begin{aligned}
G_{s}(f) & =\frac{|Y(f)|^{2}}{T_{c}} \\
& =\frac{1}{\pi^{2} f^{2} T_{c}} \sum_{i=1}^{M} \sum_{k=1}^{M}(-1)^{i+k} \sin \left(\beta_{i} f\right) \sin \left(\beta_{k} f\right) \cos \left(\gamma_{i k} f\right),
\end{aligned}
$$

with $\beta_{i} \equiv \pi T_{i}$ and $\gamma_{i k} \equiv 2 \pi\left(\bar{t}_{i}-\bar{t}_{k}\right)$.

Let $y_{1}(t)$ and $y_{2}(t)$ denote the waveforms $y(t)$ with parameters $a$ and $2-a$, respectively. It can be shown that $y_{2}(t)= \pm y_{1}\left(T_{c}-t\right)$, for $0 \leq t \leq T_{c}$, and the Fourier transforms of the two waveforms are related by $Y_{2}(f)= \pm \exp \left(-j 2 \pi f T_{c}\right) Y_{1}^{*}(f)$. As a consequence, signals $s(t)$ using waveforms $y(t)$ with parameters $a$ and $2-a$, for $0 \leq a \leq 2$, have the same PSD and autocorrelation functions.

Figure 3 displays the normalized PSD of $\operatorname{GBOCs}(6,1,0)$, $\operatorname{BOCs}(6,1), \operatorname{CBOCc}(6,1,0)$, and $\operatorname{BOCc}(6,1)$ schemes. Note that, whereas the local maxima of the power spectrum of $\operatorname{BOCs}(6,1)$ and $\operatorname{BOCc}(6,1)$ are concentrated in a small number of frequencies, the spectra of the $\operatorname{GBOCs}(6,1,0)$ and $\mathrm{CBOCc}(6,1,0)$ schemes are more regularly distributed in the frequency domain, especially in the band $|f| \leq 12.5$ MHz. This feature has a positive impact on the robustness of the receiver to narrowband interference, as will be discussed later.

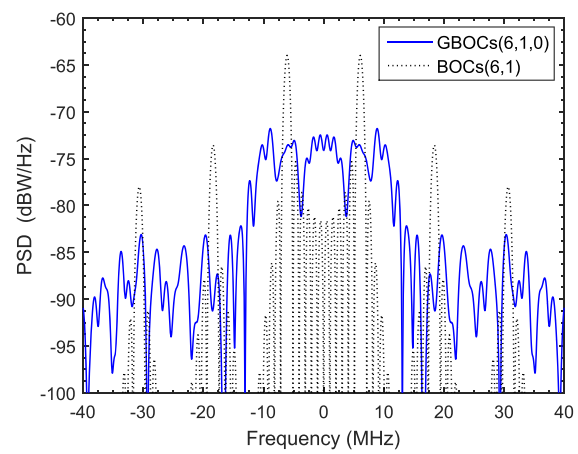

The fractional out-of-band powers (FOBP) versus the baseband bandwidth $B$ are plotted in Figure 4 for different $\operatorname{GBOCs}(m, 1, a)$ and $\operatorname{GBOCc}(m, 1, a)$ modulations. It is shown that the GBOCc schemes exhibit slightly greater FOBP values than the GBOCs counterparts. For optimal performance the sampling rates $r_{s}$ of the baseband signals at the transmitter and receiver should verify $r_{s} \geq 2 B$, where $B$ is the bandwidth corresponding to the maximum admissible value of FOBP.

The bandlimited autocorrelation function of signal $s(t)$ is ${ }^{2}$

$$
\begin{aligned}
\widetilde{R}_{S}(\tau) & =\int_{-B}^{B} G_{s}(f) \exp (j 2 \pi f \tau) d f \\
& =\frac{1}{\pi^{2} T_{c}} \sum_{i=1}^{M} \sum_{k=1}^{M}(-1)^{i+k} I\left(\beta_{i}, \beta_{k}, \gamma_{i k}, \mu(\tau) ; B\right),
\end{aligned}
$$

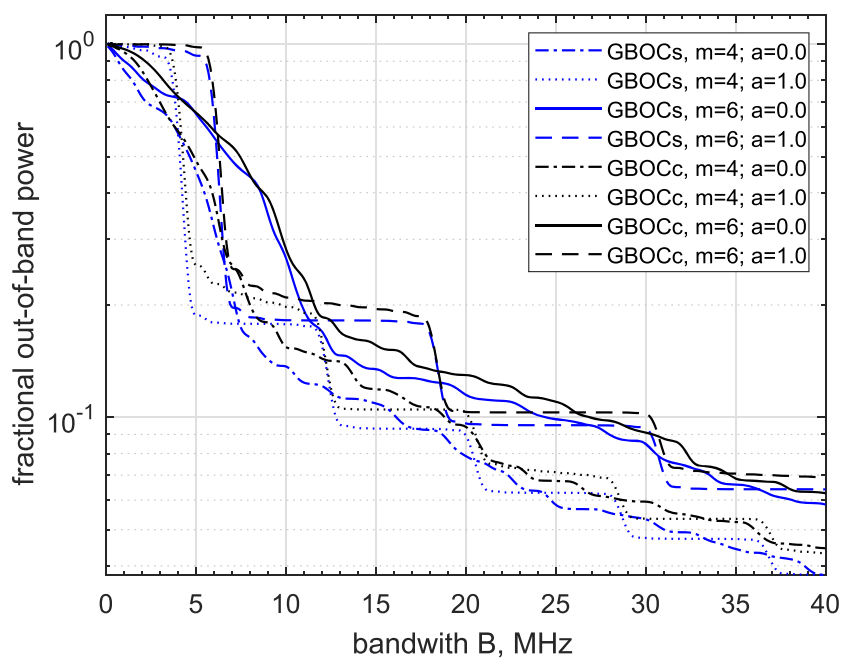

FIGURE 4 Fractional out-of-band powers for $\operatorname{GBOC}(m, 1, a)$ schemes versus the bandwidth $B$ [Color figure can be viewed at wileyonlinelibrary.com and www.ion.org]

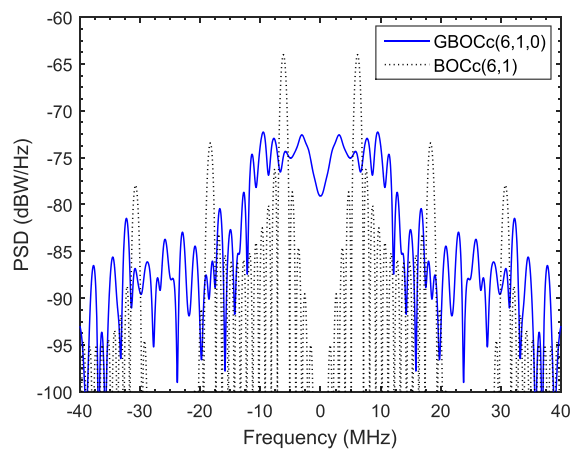

FIGURE 3 Normalized PSD of GBOCs $(6,1, a)$ and $\operatorname{GBOCc}(6,1, a)$ schemes with $a=0$ and $a=1$ (BOCs or BOCc) [Color figure can be viewed at wileyonlinelibrary.com and www.ion.org] 
where $B$ is the baseband signal bandwidth and $\mu(\tau) \equiv 2 \pi \tau$. It can be shown that

$$
\begin{aligned}
I\left(\beta_{i}, \beta_{k}, \gamma_{i k}, \mu ; B\right) & \equiv \int_{-B}^{B} \frac{\sin \left(\beta_{i} f\right) \sin \left(\beta_{k} f\right) \cos \left(\gamma_{i k} f\right) \cos (\mu f)}{f^{2}} d f \\
& =\frac{1}{4} \sum_{s=1}^{2} \sum_{r=1}^{2} \sum_{q=1}^{2}(-1)^{s+1} \int_{0}^{B} \frac{\cos [L(q, r, s) f]}{f^{2}} d f,
\end{aligned}
$$

where

$$
L(q, r, s)=\beta_{i}+(-1)^{s} \beta_{k}+(-1)^{r} \gamma_{i k}+(-1)^{q} \mu,
$$

and

$$
\int \frac{\cos (v x)}{x^{2}} d x=-\frac{\cos (v x)}{x}-v \operatorname{Si}(v x),
$$

with $\operatorname{Si}(\cdot)$ denoting the sine integral function defined $b^{7}$

$$
\operatorname{Si}(z)=\int_{0}^{z} \frac{\sin t}{t} d t
$$

Taking into account that $\lim _{x \rightarrow 0}[\cos (u x)-\cos (v x)] / x=0$ yields

$$
\begin{aligned}
I\left(\beta_{i}, \beta_{k}, \gamma_{i k}, \mu ; B\right)= & \frac{1}{4} \sum_{s=1}^{2} \sum_{r=1}^{2} \sum_{q=1}^{2}(-1)^{s}\left\{\frac{\cos [L(q, r, s) B]}{B}\right. \\
& +L(q, r, s) \operatorname{Si}[L(q, r, s) B]\}
\end{aligned}
$$

and

$$
\lim _{B \rightarrow \infty} I\left(\beta_{i}, \beta_{k}, \gamma_{i k}, \mu ; B\right)=\frac{\pi}{8} \sum_{s=1}^{2} \sum_{r=1}^{2} \sum_{q=1}^{2}(-1)^{s}|L(q, r, s)| .
$$

Figure 5 presents the normalized autocorrelation functions for several $\operatorname{GBOCs}(6,1, a)$ and $\operatorname{GBOCc}(6,1, a)$ schemes with $B=12.5 \mathrm{MHz}$. Notice that the main lobe is practically independent of the value of $a$ but the magnitude of the secondary lobes increases as $a$ approaches one. The fact that the magnitude of the secondary lobes is small for
$0 \leq a \leq 0.5$ (or $1.5 \leq a \leq 2$ ) is important both during the code acquisition (as the probability of convergence to the incorrect code phase is minimized) and in tracking mode (because the probability of false code-locking is reduced).

The block diagram for generating a GBOC signal is shown in Figure 6 being similar to the generation of the conventional BOC signal (see, for instance, Betz ${ }^{2}$ ). The main difference is that the GBOC waveform generator is not, in general, a square wave generator. By the same token, the GBOC receiver has a similar structure to the conventional BOC receiver with the only important difference being the local GBOC waveform generator that replaces the square wave generator.

\section{3 | PERFORMANCE EVALUATION}

Several aspects can be envisaged regarding the performance evaluation of the proposed GBOC schemes. GBOC is compared with other modulations in terms of spectral occupancy and Gabor bandwidth, and the Doppler effect is discussed. Next, the noncoherent early-late processing (NELP) code discriminator is used to analyze the robustness to false code-locks, evaluate the performance

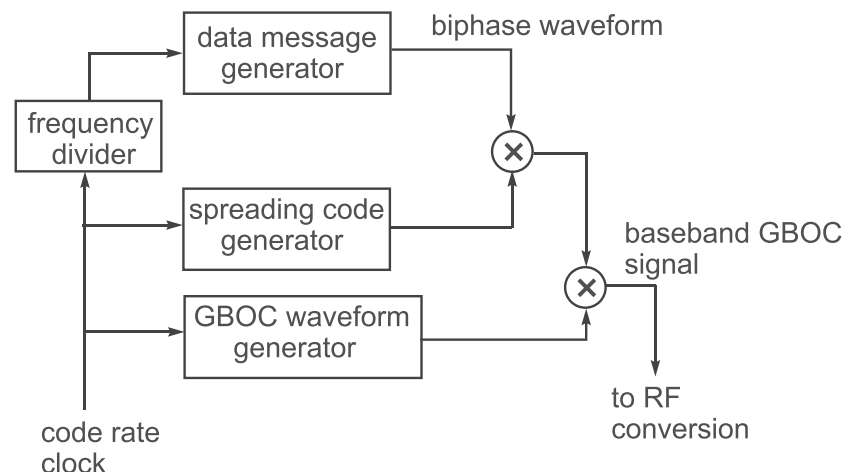

FIGURE 6 GBOC signal generation

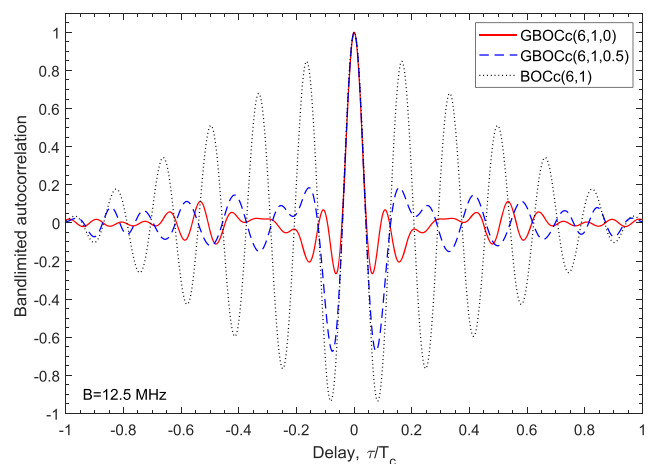

FIGURE 5 Normalized autocorrelation functions for $\operatorname{GBOC}(6,1, a)$ schemes with $a=0,0.5$ and 1 using bandwidth $B=12.5 \mathrm{MHz}$ [Color figure can be viewed at wileyonlinelibrary.com and www.ion.org] 
in white noise and multipath scenarios, and determine the robustness to narrowband and wideband interference (jamming).

\section{1 | Performance versus the bandwidth}

In positioning problems, the range accuracy depends on the channel noise and signal power spectra. By resorting to the Cramér-Rao lower bound (CRLB), it can be shown that the variance of the transmitter/receiver propagation delay estimate $\left(\widehat{\tau}_{0}\right)$ of a received baseband signal $s(t)$ of bandwidth $B$ ( $2 B$ is the corresponding front-end bandwidth) observed in the interval $[0, T]$ in the presence of additive white Gaussian noise (AWGN) with PSD $N_{0} / 2$ is given by Kay and Spilker ${ }^{8,9}$

$$
\operatorname{var}\left(\widehat{\tau}_{0}\right) \geq \frac{N_{0}}{2 \int_{0}^{T}(d s / d t)^{2} d t} .
$$

Alternatively, the CRLB for the delay estimate may be determined from $\operatorname{var}\left(\hat{\tau}_{0}\right) \geq N_{0} /\left(2 E_{S} \overline{F^{2}}\right)$, with $E_{S}$ denoting the energy of $s(t)$ and the signal mean square bandwidth (MSB) being given by Kay and Spilker ${ }^{8,9}$

$$
\overline{F^{2}}=\frac{\int_{-B}^{B}(2 \pi f)^{2}|S(f)|^{2} d f}{\int_{-B}^{B}|S(f)|^{2} d f} .
$$

This means that signals with large values of MSB are highly desirable in positioning systems and signals with large values of $|S(f)|$ at frequencies with $0 \ll|f| \leq B$ are good candidates. Taking into account (6) and (8), the MSB may be expressed as

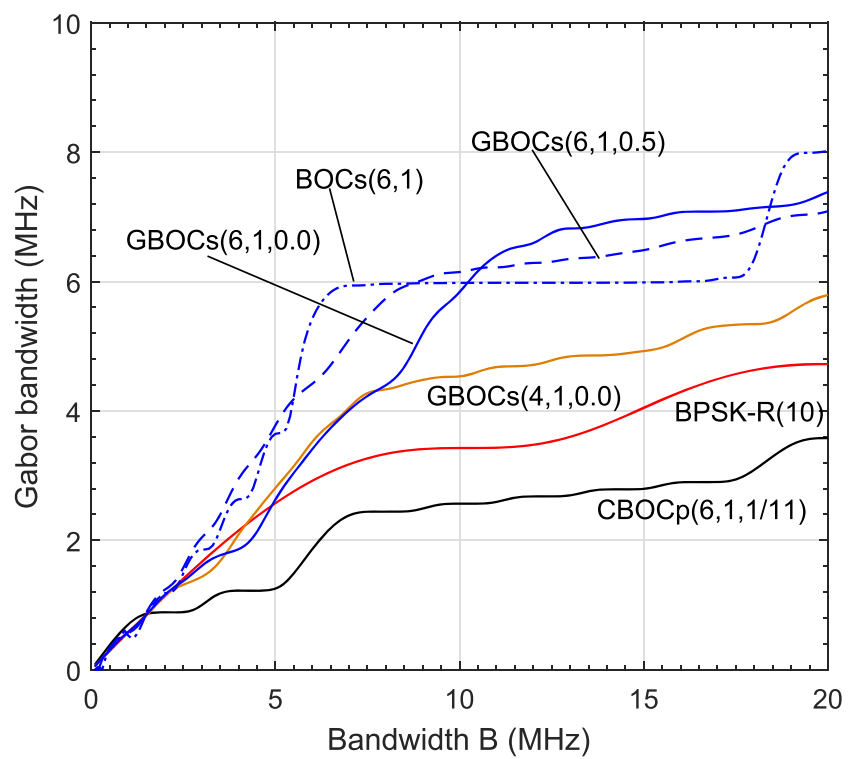

FIGURE 7 Comparison of Gabor bandwidths for GBOCs, CBOCp $(6,1,1 / 11)$ and BPSK-R(10) schemes versus the bandwidth $B$ [Color figure can be viewed at wileyonlinelibrary.com and www.ion.org]

Figure 7 displays the square root of MSB or Gabor bandwidth (GB), $\sqrt{\overline{F^{2}}} /(2 \pi)$, in $\mathrm{Hz}$ for a $\operatorname{CBOC}(6,1,1 / 11)$ pilot used in Galileo, ${ }^{10}$ BPSK-R(10), and various GBOCs schemes versus the RF bandwidth.

The PSD of the $\operatorname{CBOCp}(6,1,1 / 11)$ (pilot) signal is given by Sousa ${ }^{11}$

$$
G_{s}(f)=\frac{T_{c 0}}{11} \operatorname{sinc}^{2}\left(f T_{c 0}\right)\left[10 \tan ^{2}\left(\frac{\pi f T_{c 0}}{2}\right)+\tan ^{2}\left(\frac{\pi f T_{c 0}}{12}\right)\right]-\frac{\sqrt{10} T_{c 0}}{198} \operatorname{sinc}^{2}\left(\frac{f T_{c 0}}{12}\right) \frac{\sin ^{2}\left(\pi f T_{c 0} / 2\right) \sin \left(\pi f T_{c 0}\right)}{\sin \left(\pi f T_{c 0} / 6\right)},
$$

$$
\overline{F^{2}}=\frac{\int_{-B}^{B}(2 \pi f)^{2} G_{s}(f) d f}{\int_{-B}^{B} G_{S}(f) d f} .
$$

The positioning accuracy obtained with a given GNSS modulation depends on the sharpness of its bandlimited autocorrelation function $\widetilde{R}_{S}(\tau)$ at $\tau=0$, which is given by the MSB according to previous studies ${ }^{1,9}$

$$
-\left.\frac{1}{\widetilde{R}_{S}(0)} \frac{d^{2} \widetilde{R}_{S}(\tau)}{d \tau^{2}}\right|_{\tau=0}=\frac{4 \pi^{2}}{\widetilde{R}_{S}(0)} \int_{-B}^{B} f^{2} G_{S}(f) d f=\overline{F^{2}}
$$

Using (8), we obtain for the GBOC modulation and the PSD of the BPSK-R(10) modulation is given by $G_{s}(f)=T_{c} \operatorname{sinc}^{2}\left(f T_{c}\right)$, with $T_{c}=T_{c 0} / 10$.

The figure shows that the CBOC pilot is less efficient than all the presented schemes except for very small bandwidths where all the modulations have approximately the same GB. It is interesting to compare the performance of the $\operatorname{GBOCs}(6,1, a)$ schemes for different values of $a$. Whereas the scheme with $a=0.0$ presents a steady growth of GB with $B$, the $\operatorname{BOCs}(6,1)=\operatorname{GBOCs}(6,1,1.0)$ exhibits an approximately stepwise increase with the bandwidth. As a consequence, the GB is larger for the GBOCs $(6,1, a)$ schemes with $a<1.0$ than for the BOCs $(6,1)$ modulation in the interval $B_{0}<B \lesssim 18 \mathrm{MHz}$

$$
\overline{F^{2}}=\frac{2 B}{T_{c} \widetilde{R}_{S}(0)} \sum_{i=1}^{M} \sum_{k=1}^{M}(-1)^{i+k} \sum_{r=1}^{2} \sum_{q=1}^{2}(-1)^{r+1} \operatorname{sinc}\left(\frac{\left[\beta_{i}+(-1)^{r} \beta_{k}+(-1)^{q} \gamma_{i k}\right] B}{\pi}\right) .
$$




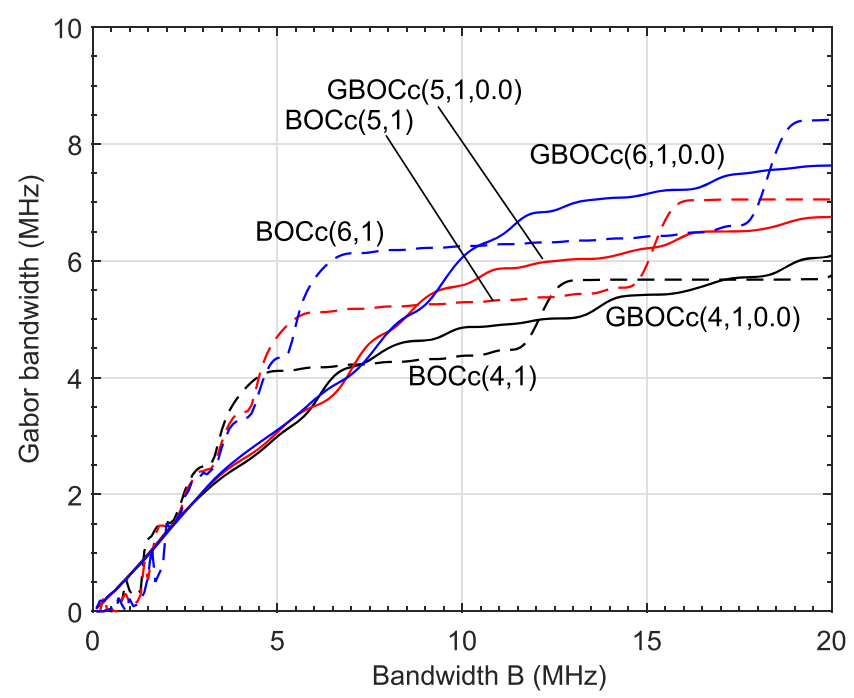

FIGURE 8 Comparison of Gabor bandwidths for GBOCc schemes versus the bandwidth $B$ [Color figure can be viewed at wileyonlinelibrary.com and www.ion.org]

with $B_{0} \lesssim 10.5 \mathrm{MHz}$. This behavior is also found in other $\operatorname{GBOCs}(m, n, a)$ schemes, with $a<1.0$, and constitutes an advantage regarding the corresponding $\operatorname{BOCs}(m, n)$ modulations.

Figure 8 presents the $\mathrm{GB}$ for $\operatorname{GBOCc}(m, 1, a)$ signals with $a=0.0$ and 1.0 versus the bandwidth $B$. Note that the curves are similar to the corresponding curves of $\operatorname{GBOCs}(m, 1, a)$ in Figure 7 although not equal. In fact, the GBOCc schemes exhibit a small increment of performance regarding the GBOCs schemes for the same bandwidth $B$. In the same way as in GBOCs schemes, there are intervals of bandwidth where the performance of $\operatorname{GBOCc}(m, 1,0.0)$ schemes exceeds that of $\operatorname{BOCc}(m, 1)$ modulations. This is true, for instance, in the interval $11 \lesssim B \lesssim 18 \mathrm{MHz}$ for $\operatorname{GBOCc}(6,1,0.0)$.

Figure 9 displays the GB of several $\operatorname{GBOC}(m, 1, a)$ schemes versus $m$ for $a=0,0.5$ and 1 with bandwidth $B=12.5 \mathrm{MHz}$. The results show that the GB increases with $m$ for $m \leq 7$ regardless of the value of parameter $a$. However, for $m>7$, there is saturation of the GB achieved with $a=0$, although it continues to increase for $a=0.5$ and 1 . The saturation for $a=0.5$ occurs at $m=9$.

\section{2 | Doppler effect}

The Doppler effect has the following consequences: (a) it changes the carrier frequency of the incoming signal from the nominal value $f_{c}$ to approximately $f_{c}(1-\Gamma)$, where $\Gamma=$ $(d r / d t) / c$, with $r$ denoting the receiver-satellite range and $c$ the speed of light, and (b) it introduces a time companding (compression/expansion) of the signal complex envelope, ${ }^{2}$ i.e., $s^{\prime}(t)=s[t(1-\Gamma)]$, where $s(t)$ is the original envelope. For conventional scenarios we typically have $|\Gamma|<10^{-5}$.

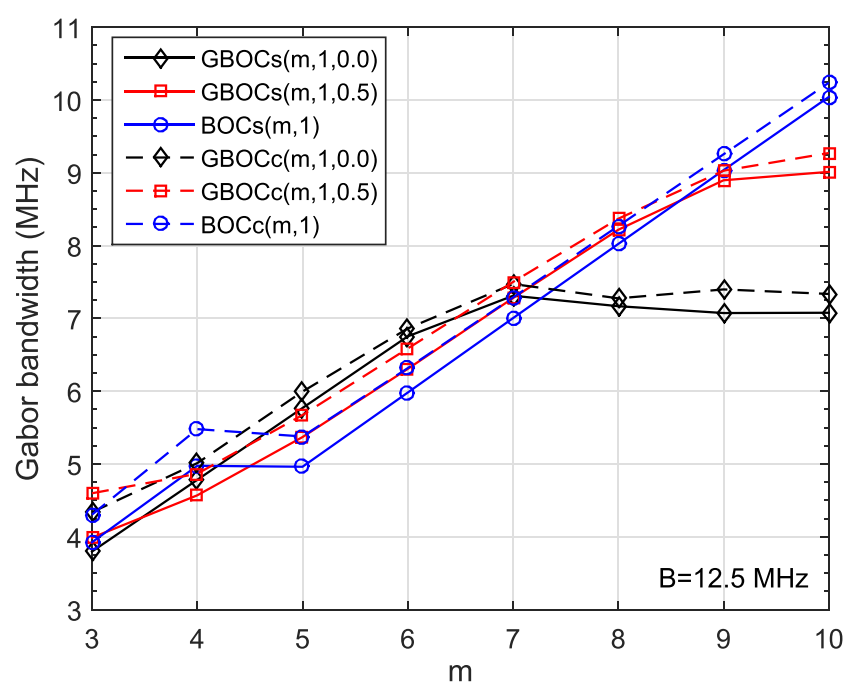

FIGURE 9 Comparison of Gabor bandwidths for $\operatorname{GBOC}(m, 1, a)$ schemes versus parameter $m$ with bandwidth $B=12.5 \mathrm{MHz}$ [Color figure can be viewed at wileyonlinelibrary.com and www.ion.org]

The cross-correlation of $s^{\prime}(t)$ and $s(t)$ is

$$
\begin{gathered}
R_{s s^{\prime}}(\Gamma)=\frac{1}{T} E\left\{\int_{0}^{T} s(t) s(t(1-\Gamma)) d t\right\} \\
=\frac{1}{N T_{c}} \sum_{k=0}^{N-1 N-1} \sum_{l=0}^{T} E\left\{c_{k} c_{l}\right\} \int_{0}^{T} y\left(t-k T_{c}\right) y\left(t(1-\Gamma)-l T_{c}\right) d t,
\end{gathered}
$$

with the signaling pulse $y(t)$ being defined in (1). Considering that $E\left\{c_{k}^{2}\right\}=1$ and $E\left\{c_{k} c_{l}\right\}=0$, with $l \neq k$, yields

$$
R_{S s^{\prime}}(\Gamma)=\frac{1}{N T_{c}} \sum_{k=0}^{N-1} \int_{k T_{c}}^{(k+1) T_{c}} y\left(t-k T_{c}\right) y\left(t(1-\Gamma)-k T_{c}\right) d t .
$$

Case one $(\Gamma<0$ : time compression):

$$
\begin{aligned}
R_{s s^{\prime}}(\Gamma) & \approx \frac{1}{N} \sum_{k=0}^{N-1} \int_{0}^{U} y(\beta) y(\beta-k \Gamma /(1-\Gamma)) d \beta, \\
U & \equiv(1+k \Gamma) /(1-\Gamma) .
\end{aligned}
$$

Case two $(\Gamma>0$ : time expansion):

$$
\begin{aligned}
R_{S s^{\prime}}(\Gamma) & \approx \frac{1}{N} \sum_{k=0}^{N-1} \int_{L}^{1} y(\beta) y(\beta-k \Gamma /(1-\Gamma)) d \beta, \\
L & \equiv k \Gamma /(1-\Gamma) .
\end{aligned}
$$

Figure 10 displays the loss of correlation versus the companding factor $\Gamma$ for different $\operatorname{GBOCs}(m, 1,0.0)$ and $\operatorname{GBOCc}(m, 1,0.0)$ modulations and different correlation intervals $T$ using a random code sequence with period equal to 1023 chips and duration of $1 \mathrm{~ms}$. We assumed no bandwidth constraints for the received signals. The results 

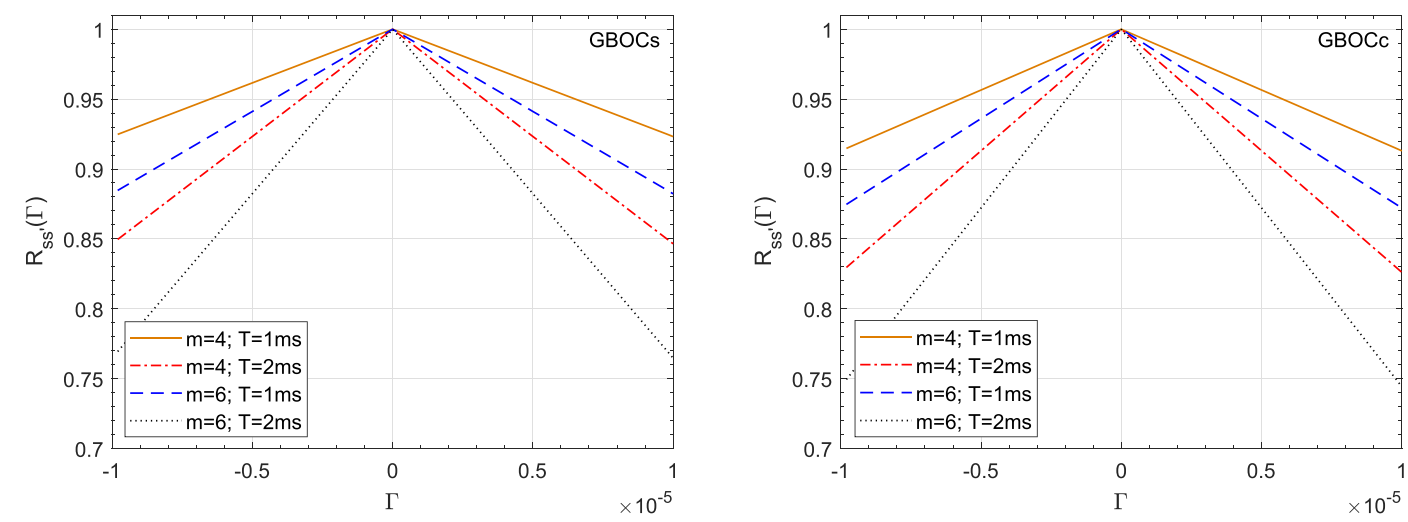

FIGURE 10 Loss of correlation versus the companding factor $\Gamma$ for GBOCs and GBOCc modulations for infinite bandwidth ( $T$ is the correlation interval) [Color figure can be viewed at wileyonlinelibrary.com and www.ion.org]
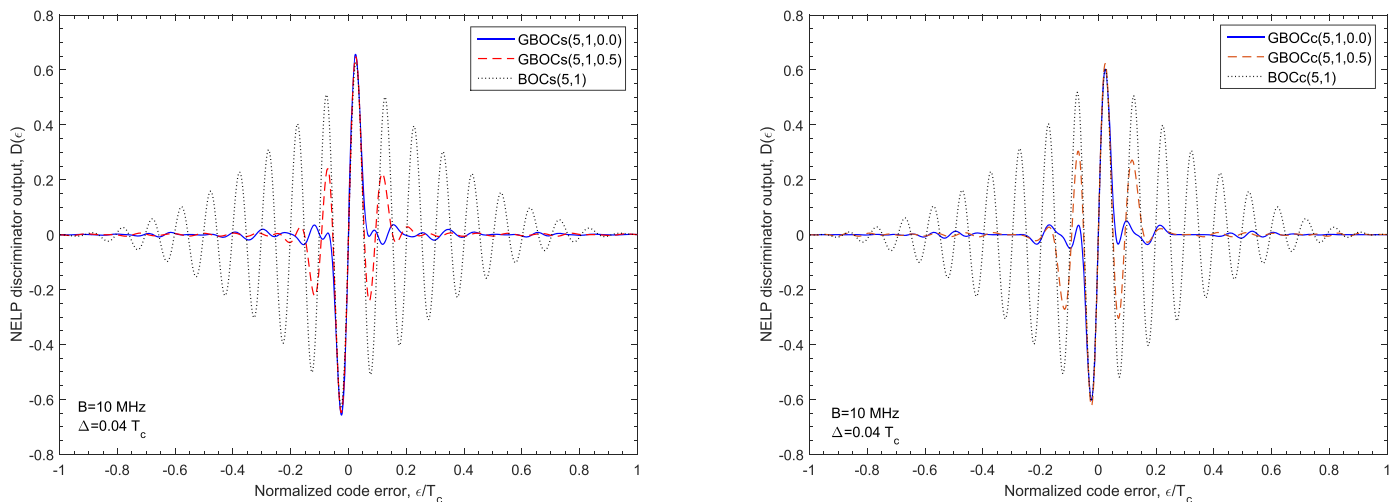

FIGURE 11 NELP discriminator S-curves for $\operatorname{GBOCs}(5,1, a)$ and $\operatorname{GBOCc}(5,1, a)$ schemes with $a=0,0.5$ and 1 using bandwidth $B=10$ $\mathrm{MHz}$ with $\rho=1$ [Color figure can be viewed at wileyonlinelibrary.com and www.ion.org]

of the two plots are similar although there is a slight advantage in using GBOCs, especially for large integration intervals. Other simulation results (not depicted) reveal that the loss of correlation is essentially independent of parameter $a$. The plots show that the loss of correlation increases with the duration of the correlation interval and with parameter $m$. This means that the loss of correlation may be prohibitive for large values of $m$ and long correlation intervals unless the time expansion/compression effect is taken into consideration in the receiver design. Note, however, that this problem is not specific to GBOC modulations and also occurs, for instance, with conventional BOC modulations. The reason for this is that the loss of correlation essentially depends on the number of transitions of $s(t)$ within the correlation interval.

\section{3 | Performance of the code discriminator}

Consider now the NELP discriminator with output (S-curve) given by Betz ${ }^{12}$

$$
D(\epsilon)=\rho\left[\widetilde{R}_{S}^{2}(\epsilon-\Delta / 2)-\widetilde{R}_{S}^{2}(\epsilon+\Delta / 2)\right],
$$

where $\rho$ depends on the power of the incoming GNSS signal, $\epsilon$ is the code tracking error and $\Delta$ is the correlators early-late spacing.

The NELP discriminator response versus the normalized code tracking error $\epsilon / T_{c}$ is shown in Figure 11 for $\operatorname{GBOCs}(5,1, a)$ and $\operatorname{GBOCc}(5,1, a)$ schemes (henceforth jointly denoted as $\operatorname{GBOC}(5,1, a))$ with $\Delta=0.04 T_{c}$ and RF bandwidth $B=10 \mathrm{MHz}$. The region of linearity around $\epsilon=0$ is approximately the same for all the modulations but the discriminator response is quite different away from the origin. For $a=0.5$ and $a=1.0$ the discriminator may achieve several stable-locking conditions for $\epsilon \neq 0$. Recall that a stable code-locking condition corresponds to $D(\epsilon)=0$ with $d D(\epsilon) / d \epsilon>0$. When there are several locking solutions the DLL is prone to tracking the code incorrectly. For $a=0.0$ there is only a stable locking point corresponding to $\epsilon=0$ with the other potential locking points being rather unstable. Two mechanisms can generate a false code-lock ${ }^{13}$ : (a) an incorrect acquisition that would acquire on a secondary peak of the autocorrelation function followed by ambiguous tracking or (a) a short loss of lock (due, for instance, to a brief decrease of $C / N_{0}$ ) followed by the lock on a secondary peak after a drift of the 
code tracking. Although several solutions have been proposed to remedy this problem, such as the bump-jumping technique, ${ }^{3,4}$ it is advantageous to operate with modulations providing code discriminator S-curves devoid of secondary peaks. Note that, for $\operatorname{GBOC}(5,1,0.0)$ schemes, the NELP discriminator S-curve approximates rather well the ideal discriminator response; ie, it is linear in an interval centered at zero and decays almost monotonously to zero outside that interval. In this case, the probability of false code-lock is virtually equal to zero for practical values of $C / N_{0}$.

The NELP discriminator gain is defined as

$$
\left.G \equiv \frac{d D}{d \epsilon}\right|_{\epsilon=0}=-\left.4 \rho \widetilde{R}_{S}(\Delta / 2) \frac{d \widetilde{R}_{S}(\epsilon)}{d \epsilon}\right|_{\epsilon=\Delta / 2},
$$

with

$$
\frac{d \widetilde{R}_{s}(\epsilon)}{d \epsilon}=\frac{2}{\pi T_{c}} \sum_{i=1}^{M} \sum_{k=1}^{M}(-1)^{i+k} \frac{\partial I\left(\beta_{i}, \beta_{k}, \gamma_{i k}, \mu ; B\right)}{\partial \mu},
$$

and

$$
\frac{\partial I\left(\beta_{i}, \beta_{k}, \gamma_{i k}, \mu ; B\right)}{\partial \mu}=\frac{1}{4} \sum_{s=1}^{2} \sum_{r=1}^{2} \sum_{q=1}^{2}(-1)^{r+s} \operatorname{Si}[L(q, r, s) B] .
$$

Figure 12 displays the NELP discriminator gains $G$ for $\operatorname{GBOCs}(m, 1,0.0)$ schemes with $m=3, \ldots, 8$ versus the normalized early-late spacing $\Delta / T_{c}$ for $B=12.5 \mathrm{MHz}$. Each curve shows an increase with $\Delta$ followed by a decay for large values of $\Delta$. As $m$ grows, the maximum gain

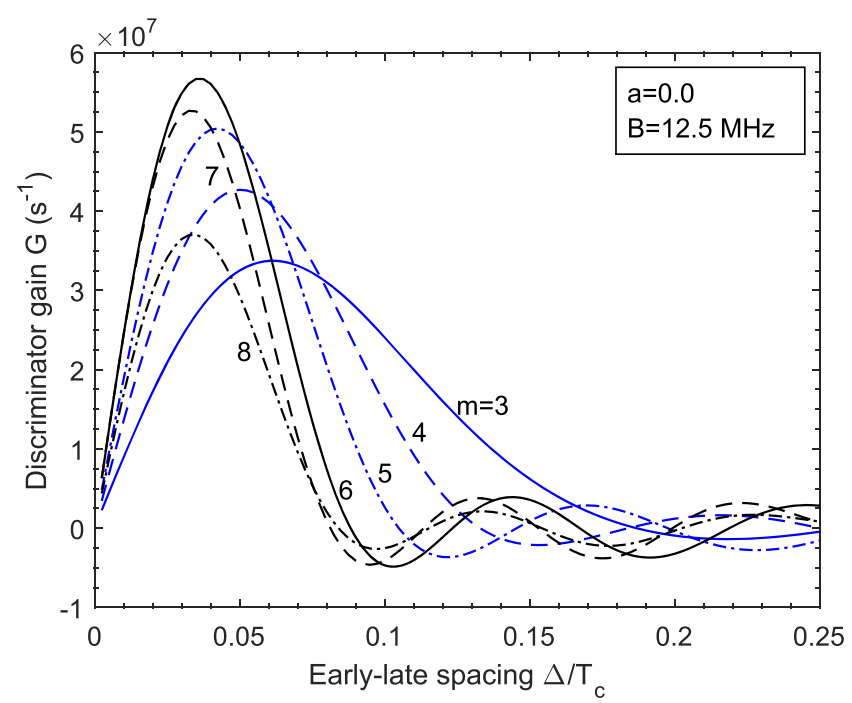

FIGURE 12 NELP discriminator gains for $\operatorname{GBOCs}(m, 1,0.0)$ schemes versus the normalized early-late spacing for $B=12.5$ $\mathrm{MHz}$ with $\rho=1$ [Color figure can be viewed at wileyonlinelibrary.com and www.ion.org] is achieved at smaller values of $\Delta$ (with typically $\Delta<$ $0.05 T_{c}$ ). The maximum value of $G$ increases with $m$ up to $m=6$, then exhibits a drop for larger values of $m$ which is due to the limited value of bandwidth $B$. Similar results are obtained with the $\operatorname{GBOCc}(m, 1,0.0)$ schemes. Therefore, $m=6$ is a good design parameter for GBOC schemes with bandwidth $B=12.5 \mathrm{MHz}$.

\section{4 | Robustness to false code-locks}

Let the baseband version of the received signal be given by

$$
\begin{aligned}
z(t)= & \sum_{i=1}^{N_{s}} \sqrt{2 P_{r}^{(i)}} d^{(i)}\left(t-\tau^{(i)}\right) S^{(i)}\left(t-\tau^{(i)}\right) \\
& \exp \left[j\left(\omega_{d}^{(i)} t+\theta^{(i)}\right)\right]+n(t),
\end{aligned}
$$

where $N_{s}$ is the number of satellites in view, $P_{r}^{(i)}$ is the power of the incoming GNSS signal $i, s^{(i)}(t)$ is defined in $(5), d^{(i)}(t)$ is the data signal, $\tau^{(i)}$ is the propagation delay, $\omega_{d}^{(i)}$ is the Doppler frequency and $n(t)$ is complex Gaussian noise. For the sake of simplicity, we remove the signal index $i$ in the subsequent equations. Consider that for each signal the code/frequency acquisition procedure consists of computing the ambiguity function ${ }^{14}$

$$
\chi\left(\tau_{e}, \omega_{e}\right)=\frac{1}{T} \int_{0}^{T} z(t) s(t-\hat{\tau}) \exp \left(-j \widehat{\omega}_{d} t\right) d t, \quad i=1, \ldots, N_{s},
$$

for a set of values $\left(\widehat{\tau}, \widehat{\omega}_{d}\right)$ of the search area, with $\tau_{e}=\hat{\tau}-\tau$ and $\omega_{e}=\widehat{\omega}_{d}-\omega_{d}$ denoting, respectively, the code delay and Doppler frequency errors. The code delay/frequency cell of the search area is selected by maximizing $\left|\chi\left(\tau_{e}, \omega_{e}\right)\right|^{2}$ over the set of values $\left(\hat{\tau}, \widehat{\omega}_{d}\right)$. Assuming that, in the correlation interval, the data bit is constant $(d(t)=d)$, the ambiguity function $\chi\left(\tau_{e}, \omega_{e}\right)$ is a complex Gaussian distributed random variable with mean

$$
E\left\{\chi\left(\tau_{e}, \omega_{e}\right)\right\}=\sqrt{2 P_{r}} d \exp \left[j\left(\pi f_{e} T+\theta\right)\right] R_{s}\left(\tau_{e}\right) \operatorname{sinc}\left(f_{e} T\right) .
$$

Therefore, $\left|\chi\left(\tau_{e}, \omega_{e}\right)\right|^{2}$ is a noncentral chi-square distributed random variable with two degrees of freedom. An incorrect acquisition occurs whenever an incorrect cell in the search area is selected. In that case, when the receiver switches from acquisition to tracking mode, the code tracking loop may lock or not in the incorrect code delay (false code-lock). The evaluation of the probability of a false-code lock when changing from acquisition to tracking mode is a complicated task. Instead, we consider a performance metric based on the ratio

$$
Q\left(\tau_{m}\right)=\frac{\left(E\left\{\chi\left(\tau_{m}, 0\right)\right\}\right)^{2}}{(E\{\chi(0,0)\})^{2}}=R_{S}^{2}\left(\tau_{m}\right)
$$



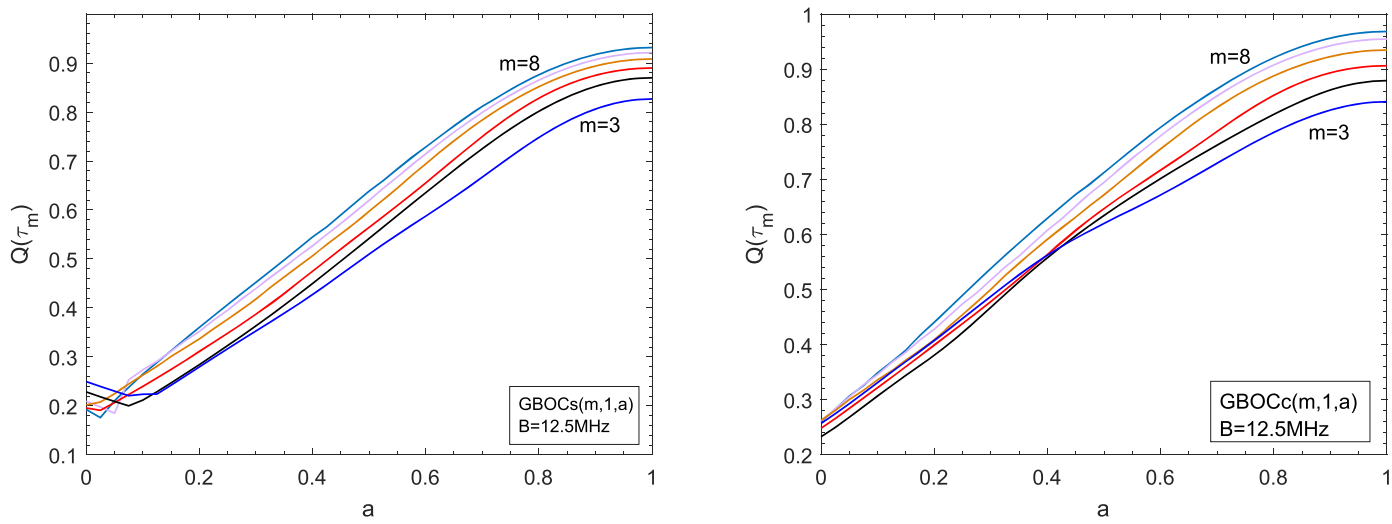

FIGURE 13 Performance metrics $Q\left(\tau_{m}\right)$ for GBOCs and GBOCc modulations with $B=12.5 \mathrm{MHz}$ [Color figure can be viewed at wileyonlinelibrary.com and www.ion.org]

where $\tau_{m}$ is the value of the code discriminator tracking error that yields the most likely stable false code-locking condition. That is, among the different values of the code tracking error that verify the stable code locking condition, we choose $\tau_{m}$ as the value of $\tau_{e}$ that maximizes $R_{s}^{2}\left(\tau_{e}\right)$. Note that the value of $\tau_{m}$ depends on the selected code discriminator. Figure 13 displays the plots of $Q\left(\tau_{m}\right)$ for various GBOCs and GBOCc modulations with $B=12.5 \mathrm{MHz}$ assuming a NELP discriminator. Since the robustness to false code-locks depends inversely on the ratio $Q\left(\tau_{m}\right)$, the best modulations are characterized by small values of $a$ (or equivalently values near 2). The plots show that the difference of performance between GBOCs and GBOCc is small although the former modulations are slightly better. Notice also that the worse performance corresponds to the BOCs and BOCc (that is, $a=1$ ) with $m$ large where $Q\left(\tau_{m}\right)$ approaches one.

\section{5 | Performance in white noise}

In Betz, ${ }^{12}$ it is shown that, for the AWGN channel, the code-tracking error variance in square meters using a delay-lock loop with NELP processing is given by

$$
\sigma_{N E L P}^{2}=\zeta \frac{J_{0}\left(\frac{C}{N_{0}}\right)^{-1}}{J_{2}^{2}}\left[1+\frac{1}{T} \frac{J_{1}\left(\frac{C}{N_{0}}\right)^{-1}}{J_{3}^{2}}\right]\left(\mathrm{m}^{2}\right),
$$

with

$$
\begin{gathered}
\zeta=\frac{c^{2} B_{L}\left(1-0.5 B_{L} T\right)}{(2 \pi)^{2}}, \\
{\left[\begin{array}{l}
J_{0} \\
J_{1}
\end{array}\right]=\int_{-B}^{B} G_{S}(f)\left[\begin{array}{l}
\sin ^{2}(\pi f \Delta) \\
\cos ^{2}(\pi f \Delta)
\end{array}\right] d f,} \\
J_{2}=\int_{-B}^{B} f G_{S}(f) \sin (\pi f \Delta) d f,
\end{gathered}
$$

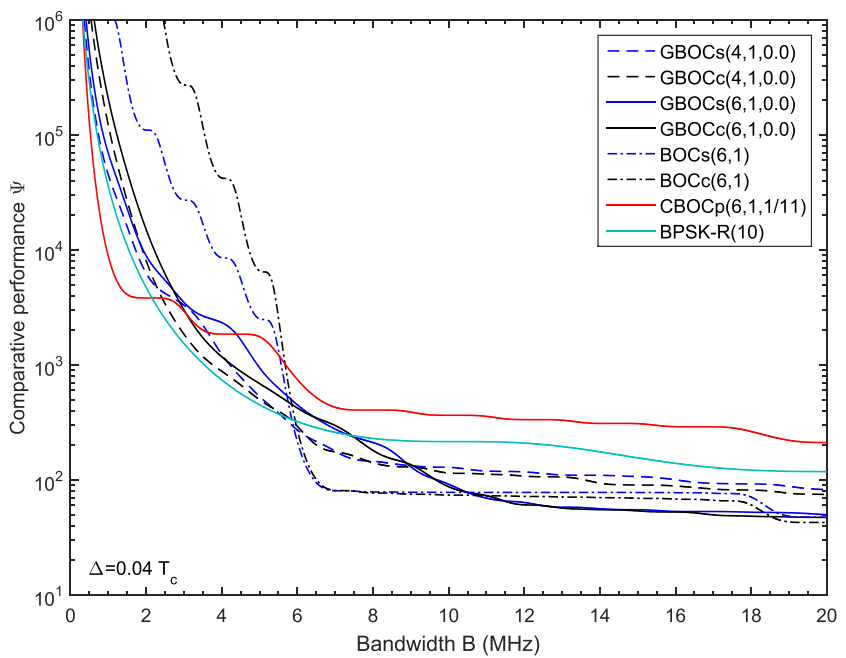

FIGURE 14 Comparative performance of the NELP receiver in AWGN using $\operatorname{GBOC}(6,1, a)$ schemes. $\operatorname{CBOCp}(6,1,1 / 11)$ and BPSK-R(10) are also included for comparison [Color figure can be viewed at wileyonlinelibrary.com and www.ion.org]

$$
J_{3}=\int_{-B}^{B} G_{S}(f) \cos (\pi f \Delta) d f .
$$

Here, $C / N_{0}$ is the carrier power to noise power spectral density ratio, $T$ is the correlation interval, and the quantity inside the square brackets in (35) is the squaring loss term of the noncoherent processing. For large values of $C / N_{0}$, a unity squaring loss is obtained, and the NELP error variance is well approximated by $\sigma_{N E L P}^{2}=\zeta J_{0}\left(C / N_{0}\right)^{-1} / J_{2}^{2}$. In those cases, the NELP error variance can be computed by multiplying $B_{L}\left(1-0.5 B_{L} T\right)\left(C / N_{0}\right)^{-1}$ by the factor $\Psi \equiv$ $c^{2} J_{0} /\left(2 \pi J_{2}\right)^{2}$ that depends only on the PSD of the modulation scheme and the NELP discriminator early-late spacing. Figure 14 displays the factor $\Psi$ versus the bandwidth $B$ for $\operatorname{GBOCs}(m, 1, a), \operatorname{GBOCc}(m, 1, a), \operatorname{CBOCp}(6,1,1 / 11)$, 
and BPSK-R(10), with $\Delta=0.04 T_{c}$, which permits comparison of the performance between different schemes. The results show that $\Psi$ decreases when the bandwidth grows, according to the Gabor bandwidth performance of Figures 7 and 8. In agreement with those results, the performance of the $\operatorname{GBOC}(6,1,0)$ schemes exceeds that of BOC $(6,1)$ schemes in the interval $10.5<B<18.5 \mathrm{MHz}$. Moreover, for $B<5.9 \mathrm{MHz}$, and unlike what is predicted by the Gabor bandwidth, the $\operatorname{GBOC}(6,1,0)$ schemes are also better than their BOC counterparts. This shows that the NELP discriminator more inefficiently processes the BOC signals when small bandwidths are used. In contrast, the GBOC signals with $a=0$ are conveniently processed. Also, for $B>6 \mathrm{MHz}$ the $\operatorname{GBOC}(6,1, a)$ schemes outperform the CBOCp $(6,1,1 / 11)$ and BPSK-R(10) modulations. This result proves that the NELP discriminator is suitable to process GBOCs signals affected by AWGN. The main advantage of the $\operatorname{GBOC}(6,1,0.0)$ modulations relative to other schemes is that the NELP discriminator tracks the spreading code with a negligible probability of false code-locks for typical values of $C / N_{0}$.

\section{6 | Multipath mitigation}

The usual way to characterize the static multipath effect with one reflected ray is to determine the multipath error envelope, which gives the maximum code tracking errors (for inphase and out-of-phase reflected rays) obtained with a given code discriminator (in our case, the NELP discriminator) for each value of the reflected ray extra delay (excess delay).

The benefits achieved with the proposed modulations in multipath channels are evidenced in Figure 15, which compares the multipath error envelopes for GBOC schemes with a bandwidth $B=12.5 \mathrm{MHz}$. The curves were obtained with the NELP discriminator using

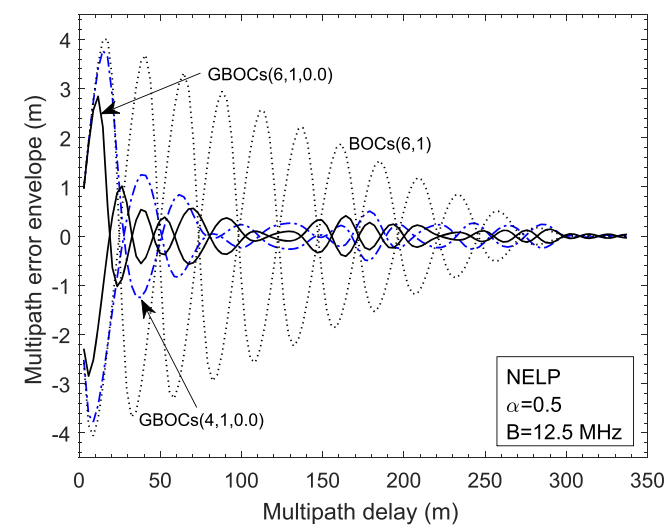

early-late spacing $\Delta=0.04 T_{c}$ and correspond to solving the equation

$$
\begin{aligned}
& {\left[\widetilde{R}_{S}(\epsilon-\Delta / 2) \pm \alpha \widetilde{R}_{S}(\epsilon-\tau-\Delta / 2)\right]^{2}} \\
& -\left[\widetilde{R}_{S}(\epsilon+\Delta / 2) \pm \alpha \widetilde{R}_{S}(\epsilon-\tau+\Delta / 2)\right]^{2}=0
\end{aligned}
$$

for the code tracking error $\epsilon$ with different values of the multipath (excess) delay $\tau$ and the relative amplitude of the secondary ray $\alpha$ made equal to 0.5 . The bandlimited autocorrelation function $\widetilde{R}_{S}(\tau)$ for GBOCs signals is given in (9), and the solution of (40) may be computed efficiently using, for instance, the Newton method.

The plots of Figure 15 show that the proposed $\operatorname{GBOC}(m, 1,0.0)$ modulations exhibit a significantly better performance than the corresponding $\operatorname{BOCs}(m, 1)$ and $\operatorname{BOCc}(m, 1)$ schemes. In addition, there is an improvement when $m=4$ is replaced with $m=6$ for the same bandwidth. The curves for $\operatorname{GBOCs}(m, 1,0.0)$ and $\operatorname{GBOCc}(m, 1,0.0)$ are similar although there is a small advantage of using the latter schemes in scenarios with small multipath delays ( $<120$ meters $)$.

\section{7 | Robustness to interference}

This subsection aims to assess the robustness of the GBOC schemes to narrowband and wideband radio-frequency interference. Often, narrowband interference signals are generated unintentionally by electronic equipment operating imperfectly. In contrast, wideband interference is frequently intentional and aims to hinder a navigation service by masking GNSS signals with interference. ${ }^{15}$

In Betz, ${ }^{12}$ expressions were also derived for the variance of the code tracking error with NELP processing in the presence of a narrowband Gaussian interference signal with baseband equivalent normalized PSD $G_{I}(f)$ and

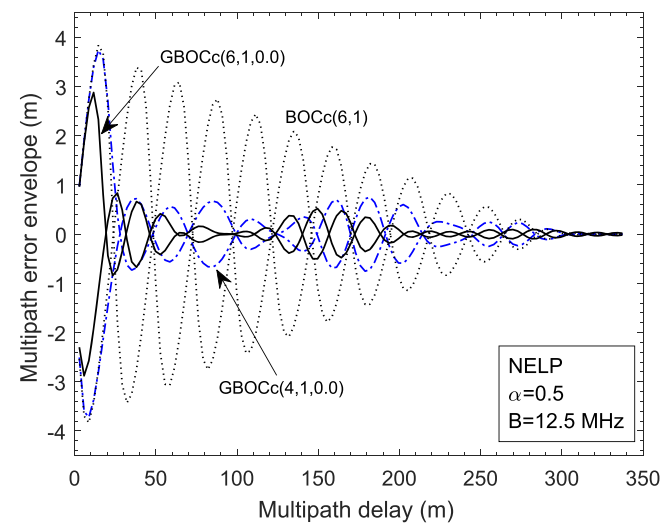

FIGURE 15 Multipath error envelopes for GBOCs and GBOCc schemes using the NELP code discriminator with $\Delta=0.04 T_{c}, \alpha=0.5$ and $B=12.5 \mathrm{MHz}$ [Color figure can be viewed at wileyonlinelibrary.com and www.ion.org] 
interference to carrier power ratio $I / C$. The resulting variance of the code tracking error encompassing AWGN and interference is given by

$$
\begin{aligned}
\sigma_{\mathrm{NELP}}^{2}= & \zeta \frac{J_{0}\left(\frac{C}{N_{0}}\right)^{-1}+\left(\frac{I}{C}\right) J_{4}}{J_{2}^{2}} \\
& {\left[1+\frac{1}{T} \frac{J_{1}\left(\frac{C}{N_{0}}\right)^{-1}+\left(\frac{I}{C}\right) J_{5}}{J_{3}^{2}}\right]\left(\mathrm{m}^{2}\right), }
\end{aligned}
$$

with $\zeta$ and parameters $J_{i}, i=0, \ldots, 3$, defined in (36) to (39), and

$$
\left[\begin{array}{l}
J_{4} \\
J_{5}
\end{array}\right]=\int_{-B}^{B} G_{I}(f) G_{S}(f)\left[\begin{array}{l}
\sin ^{2}(\pi f \Delta) \\
\cos ^{2}(\pi f \Delta)
\end{array}\right] d f .
$$

When $I=0$ (absence of interference) expression (35) is recovered. For a given value of the interference power $I$, the tracking error depends on $J_{4}$ and $J_{5}$. Expression (42) shows that the degradation imposed by the interference is more significant when its power spectrum $G_{I}(f)$ is centered at a local maximum of the modulated signal spectrum $G_{S}(f)$. This scenario typically occurs with the BOC modulations, which have spiked power spectra. In contrast, the GBOC modulations with $a \approx 0$ have relatively smooth spectra (see Figure 3), which increases their robustness to narrowband interference.

Figure 16 exhibits the root means square code tracking errors versus the offset frequency of the narrowband interference for $\operatorname{GBOCs}(m, 1, a)$ schemes. The $\mathrm{RF}$ interference power spectrum is assumed to be constant in a bandwidth of $B_{I}=10 \mathrm{kHz}$ centered at frequencies $f_{c} \pm f_{I}$ ( $f_{I}$ is the offset frequency indicated in the figure).

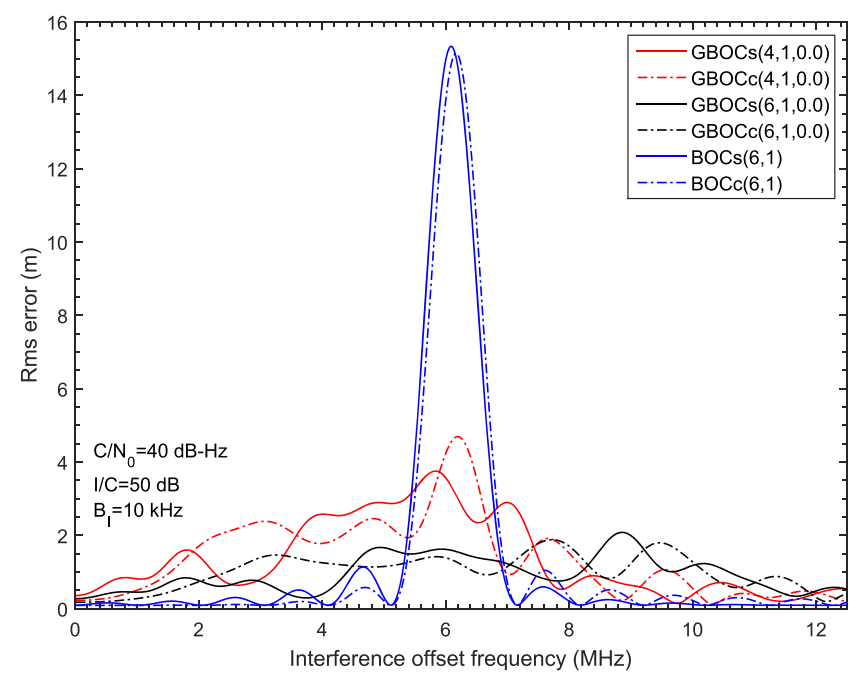

FIGURE 16 Root mean square errors versus offset frequency of the narrowband interference for different GBOC schemes [Color figure can be viewed at wileyonlinelibrary.com and www.ion.org]
The interference to signal power ratio is made equal to $I / C=50 \mathrm{~dB}$, and the carrier power to noise-density ratio is $C / N_{0}=40 \mathrm{~dB}-\mathrm{Hz}$. The DLL bandwidth is $B_{L}=1 \mathrm{~Hz}$ and the correlation interval is $T=1 \mathrm{~ms}$. The plots show that the robustness to narrowband interference increases with the modulation parameter $m$. In fact, better results are obtained with $\operatorname{GBOC}(6,1,0.0)$ than with $\operatorname{GBOC}(4,1,0.0)$ schemes. In addition, the maximum root mean square error decreases substantially when GBOC schemes replace their BOC counterparts, which is justified by the spiked shape of the PSD in the BOC schemes.

Several civil jammers were tested in existing studies ${ }^{16,17}$ and the signals found were, typically, of the chirp type with periods of repetition on the order of several dozens of microseconds and frequency sweeps exceeding $20 \mathrm{MHz}$. Based on the analysis carried out therein, we model the wideband interference as a periodic chirp signal with the instantaneous frequency $f_{i}(t)$ depicted in Figure 17, where $W$ stands for the frequency sweep range, and $T_{J}$ is the sweep period of the jamming signal. We assume that the instantaneous frequency of the interference signal is periodic with period $T_{J}$, being given by $f_{i}(t)=f_{c}+\gamma t-W / 2$ in the interval $0 \leq t<T_{J}$, where $f_{c}$ is the GNSS signal carrier frequency and $\gamma=W / T_{J}$ is the frequency sweep rate. The complex envelope $u(t)$ of the normalized version of the interference signal has period $T_{J}$ and is given by $u(t)=\exp \left[j\left(\pi \gamma t^{2}-\pi+\varphi_{0}\right)\right]$ in the interval $0 \leq t<T_{J}$. Thus, it can be expanded in complex Fourier series as

$$
u(t)=\sum_{n=-\infty}^{\infty} c_{n} \exp \left(j 2 \pi n f_{0} t\right), \quad f_{0}=1 / T_{J},
$$

with coefficients $c_{n}=U_{T}\left(n f_{0}\right) / T_{J}$, where $U_{T}(f)$ is the Fourier transform of the truncated signal $u_{T}(t)=u(t)$, for $0 \leq t<T_{J}$, and $u_{T}(t)=0$, otherwise.

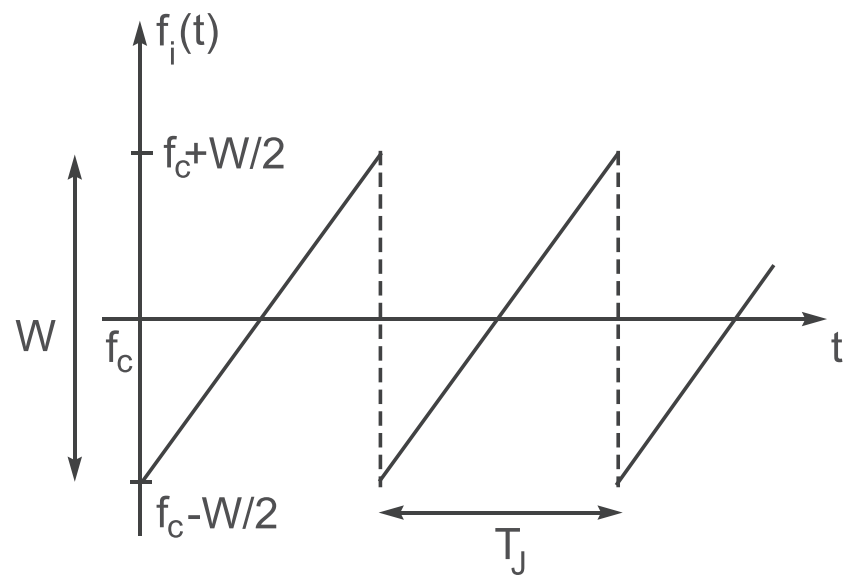

FIGURE 17 Frequency sweep of the chirp jamming signal 
The PSD of $u(t)$ is given by the Parseval theorem

$$
G_{u}(f)=\frac{1}{T_{J}^{2}} \sum_{n=-\infty}^{\infty}\left|U_{T}\left(n f_{0}\right)\right|^{2} \delta\left(f-n f_{0}\right) .
$$

It is shown in another research ${ }^{18}$ that $\left|U_{T}(f)\right|^{2}=$ $(2 \gamma)^{-1}\left\{\left[C\left(u_{2}\right)-C\left(u_{1}\right)\right]^{2}+\left[S\left(u_{2}\right)-S\left(u_{1}\right)\right]^{2}\right\}$, where $C(\cdot)$ and $S(\cdot)$ are, respectively, the cosine and sine Fresnel integrals $\left(\right.$ see $\left.^{7}\right)$ and $u_{1}(f)=-\sqrt{2 / \gamma}(f+W / 2), u_{2}(f)=$ $-\sqrt{2 / \gamma}(f-W / 2)$. Replacing (44) in (42), with $G_{I}(f)=$ $G_{u}(f)$, leads to

$$
\left[\begin{array}{l}
J_{4} \\
J_{5}
\end{array}\right]=f_{0}^{2} \sum_{n=-\infty}^{\infty}\left|U_{T}\left(n f_{0}\right)\right|^{2} G_{S}\left(n f_{0}\right)\left[\begin{array}{l}
\sin ^{2}\left(\pi n f_{0} \Delta\right) \\
\cos ^{2}\left(\pi n f_{0} \Delta\right)
\end{array}\right]
$$

Figure 18 displays the root mean square tracking errors obtained with wideband chirp interference for $\operatorname{GBOC}(m, 1, a)$ signals, where it is assumed that the front-end bandwidth is $2 B>W$, with $B=12.5 \mathrm{MHz}$, to guarantee that the chirp instantaneous frequency is in-band all the time. Otherwise, the chirp signal would be partially out-of-band leading to a pulsed interference scenario. The carrier-to-noise ratio $C / N_{0}$ and the interference-to-signal ratio $I / C$ are, respectively, made equal to $40 \mathrm{~dB}-\mathrm{Hz}$ and $50 \mathrm{~dB}$. The chirp repetition period is $T_{J}=20 \mu \mathrm{s}$. The DLL bandwidth is $B_{L}=1 \mathrm{~Hz}$, and the correlation interval is $T=1 \mathrm{~ms}$. The plots show that the robustness against wideband chirp interference increases when the modulation parameter $m$ grows. In fact, there is a decrease of the maximum root mean squares errors from approximately 2.4 to $1.4 \mathrm{~m}$ when the $\operatorname{GBOC}(4,1,0.0)$ modulations are replaced with $\operatorname{GBOC}(6,1,0.0)$. The difference

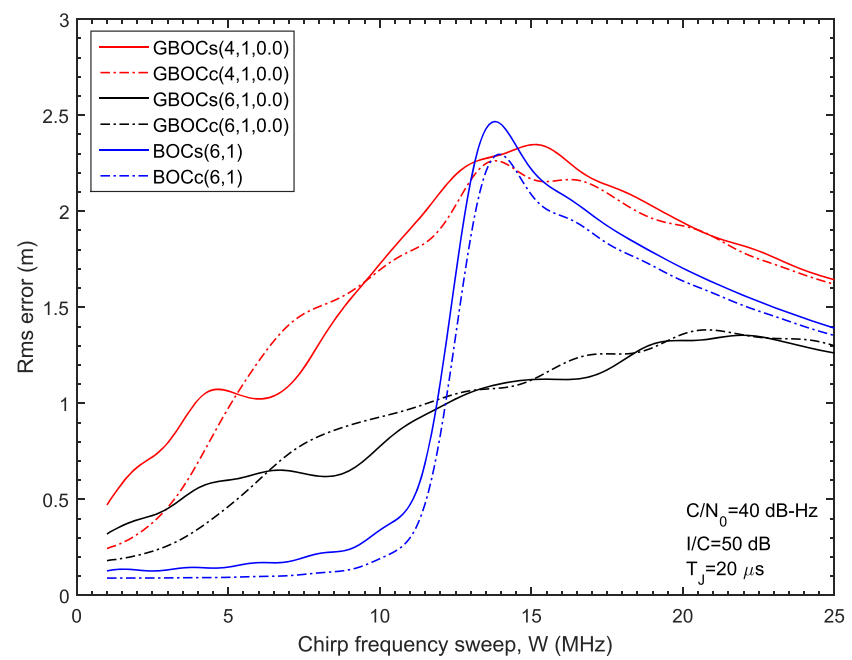

FIGURE 18 Root mean square errors versus the frequency sweep of the chirp jamming signal for $\operatorname{GBOC}(m, 1, a)$ schemes with chirp pulse duration $T_{J}=20 \mu \mathrm{s}$ [Color figure can be viewed at wileyonlinelibrary.com and www.ion.org] of performance of the modulations GBOCs and GBOCc with the same parameters is, in general, small but depends on the value of $W$ considered. The figure also shows that the $\operatorname{GBOC}(6,1,0.0)$ schemes provide more robustness to wideband interference than the corresponding $\operatorname{BOC}(6,1)$ modulations for chirp frequency sweeps higher than approximately $12 \mathrm{MHz}$.

\section{4 | CONCLUSION}

We proposed a class of digital modulation schemes for GNSS signals, named generalized binary offset carrier (GBOC), which result from replacing the square wave subcarrier of the BOC schemes with a binary signal whose fundamental frequency changes linearly within the chip interval (binary chirp waveform). The novel modulations are characterized by three parameters: $m, n$, and $a$. Parameters $m$ and $n$ jointly define the duration and number of level transitions of the chip waveform and $a$ controls the location of the transitions. It was shown that this parameter also establishes the intensity of the secondary peaks in the autocorrelation function, which take an approximately ideal triangular shape for $a=0$ or $a=2$. As a consequence, GBOC schemes with $a \approx 0$ or $a \approx$ 2 permit to minimize the probabilities of incorrect code acquisition and false code-lock in tracking mode when used in conjunction with the noncoherent early-late power code discriminator. Moreover, the performance achieved in the presence of multipath and narrowband or wideband interference exceeds that of the corresponding BOC modulations, thus suggesting the use of GBOC schemes in future GNSS applications.

\section{AKNOWLEDGMENTS}

The authors would like to thank the valuable comments of the anonymous reviewers, which helped improve the quality of the final manuscript.

This work was supported by the Portuguese national funding agency for science, research and technology (FCT) under project UID/EEA/50008/2013.

\section{ORCID}

Fernando D. Nunes (i) https://orcid.org/ 0000-0001-7864-3178

Fernando M. G. Sousa (D) https://orcid.org/ 0000-0003-2520-7553

José M. N. Leitão (iD https://orcid.org/

0000-0003-4278-0415 


\section{REFERENCES}

1. Betz JW. Binary offset carrier modulations for radionavigation. NAVIGATION. 2002;48:227-246.

2. Betz JW. Engineering Satellite-Based Navigation and Timing, Global Navigation Satellite Systems, Signals, and Receivers. Hoboken, NJ: Wiley; 2016.

3. Fine P, Wilson W. Tracking algorithm for GPS offset carrier signals. Proceedings of the 1999 National Technical Meeting of the Institute of Navigation; 1999; San Diego, CA:671-676.

4. Heiries V, Roviras D, Ries L, Calmettes V. Analysis of non ambiguous BOC signal acquisition performance. Proceedings of the 17th International Technical Meeting of the Satellite Division of the Institute of Navigation (ION GNSS 2004); 2004; Long Beach, CA:2611-2622.

5. Nunes F, Leitão J, Sousa F. Performance analysis of an absolute phase modulation scheme for GNSS signals. ESA Navitec; 2016; Noordwijk, the Netherlands:1-8.

6. Betz JW. On the power spectral density of GNSS signals, with applications. Proceedings of the 2010 International Technical Meeting of the Institute of Navigation; 2010; San Diego, CA:859-871.

7. Abramowitz M, Stegun IA, eds. Handbook of Mathematical Functions. N. York: Dover; 1970.

8. Kay S. Fundamentals of Statistical Signal Processing: Estimation Theory. Upper Saddle River: Prentice-Hall; 1993.

9. Spilker JJ. Global Positioning System: Theory and Applications. Washington DC: AIAA; 1996.

10. European, Union. European GNSS (Galileo) open service, signal in space interface control document. issue 1.2; 2015.

11. Sousa F, Nunes F. New expressions for the autocorrelation function of BOC GNSS signals. NAVIGATION. 2013;60:1-9.

12. Betz JW, Kolodzieski KR. Generalized theory of code tracking with an early-late discriminator. Part II: noncoherent processing and numerical results. IEEE Trans Aerosp Electron Syst. 2009;45(4):1551-1564.
13. Julien O, Cannon ME, Lachapelle G, Montgrédien C, Macabiau C. A new unambiguous $\mathrm{BOC}(\mathrm{n}, \mathrm{n})$ signal tracking technique. ENC-GNSS; 2004; Rotterdam, the Netherlands:1-12.

14. Misra P, Enge P. Global Positioning System, Signals, Measurements, and Performance. second. Lincoln, MA: Ganga-Jamuna; 2006.

15. Dovis F, ed. Gnss Interference Threats and Countermeasures. Boston: Artech House; 2015.

16. Kraus T, Bauernfeind R, Eissfeller B. Survey of in-car jammers analysis and modeling of the RF signals and IF samples (suitable for active signal cancellation). Proceedings of the 24th International Technical Meeting of the Satellite Division of the Institute of Navigation (ION GNSS 2011); 2011; Portland, OR:430-435.

17. Mitch R, Dougherty R, Psiaki M, et al. Signal characteristics of civil GPS jammers. Proceedings of the 24th International Technical Meeting of the Satellite Division of the Institute of Navigation ION GNSS 2011; 2011; Portland, OR:1907-1919.

18. Klauder J, Price A, Darlington S, Albersheim W. The theory and design of chirp radars. Bell Syst Tech J. 1960;39(4):745-808.

How to cite this article: Nunes FD, Sousa FMG, Leitão JMN. Characterization and performance analysis of generalized BOC modulations for GNSS. NAVIGATION. 2019;66:185-197. https://doi.org/10.1002/navi.279 